\title{
AIP
}

\section{Kinetic depinning of a magnetic domain wall above the Walker field}

E. R. Lewis, D. Petit, L. O'Brien, A.-V. Jausovec, H. T. Zeng et al.

Citation: Appl. Phys. Lett. 98, 042502 (2011); doi: 10.1063/1.3543844

View online: http://dx.doi.org/10.1063/1.3543844

View Table of Contents: http://apl.aip.org/resource/1/APPLAB/v98/i4

Published by the American Institute of Physics.

\section{Related Articles}

Domain wall propagation in micrometric wires: Limits of single domain wall regime J. Appl. Phys. 111, 07E311 (2012)

Magnetic structure and resonance properties of a hexagonal lattice of antidots Low Temp. Phys. 38, 157 (2012)

Thermally activated domain wall dynamics in a disordered magnetic nanostrip J. Appl. Phys. 109, 07D345 (2011)

Noise investigation of the orthogonal fluxgate employing alternating direct current bias

J. Appl. Phys. 109, 07E529 (2011)

Comparison of electrical techniques for magnetization dynamics measurements in micro/nanoscale structures J. Appl. Phys. 109, 07D317 (2011)

\section{Additional information on Appl. Phys. Lett.}

Journal Homepage: http://apl.aip.org/

Journal Information: http://apl.aip.org/about/about_the_journal

Top downloads: http://apl.aip.org/features/most_downloaded

Information for Authors: http://apl.aip.org/authors

\section{ADVERTISEMENT}

\section{ACCELERATE AMBER AND NAMD BY 5X.}

NVIDIA TRYIT ONA FREE, REMOTELYYHOSTED CLUSTER. 


\title{
Kinetic depinning of a magnetic domain wall above the Walker field
}

\author{
E. R. Lewis, ${ }^{1,2, a)}$ D. Petit, ${ }^{1,2}$ L. O’Brien, ${ }^{1,2}$ A.-V. Jausovec, ${ }^{1}$ H. T. Zeng, ${ }^{1}$ D. E. Read, ${ }^{1}$ and \\ R. P. Cowburn ${ }^{1,2}$ \\ ${ }^{1}$ Department of Physics, Blackett Laboratory, Imperial College London, London SW7 2BW, United Kingdom \\ ${ }^{2}$ Cavendish Laboratory, University of Cambridge, Cambridge CB3 OHE, United Kingdom
}

(Received 17 December 2010; accepted 21 December 2010; published online 24 January 2011)

\begin{abstract}
The dynamical interaction between a transverse domain wall and a T-shaped trap is investigated, for domain wall motion in the oscillatory regime above the Walker field. We demonstrate experimentally the existence of distinct static and kinetic depinning fields in this regime, and show that the oscillatory motion of the domain wall leads to a distribution of kinetic depinning fields. Micromagnetic simulations are in good qualitative agreement with our experimental results. (C) 2011 American Institute of Physics. [doi:10.1063/1.3543844]
\end{abstract}

The functioning of domain wall (DW)-based devices ${ }^{1-4}$ depends both on the characteristics of DW pinning at geometrical features which are used to control the position of the DW and on the nature of the DW motion in ferromagnetic nanostrips. Even in the absence of pinning sites, the DW motion is complicated, with a low-field viscous regime giving way to oscillatory motion above a threshold field known as the Walker field. ${ }^{5}$ The motion and the pinning of the DW are interrelated, because DW pinning depends not only on the equilibrium DW structure, but also on the dynamical state of the DW when it reaches the pinning site. This is manifested in the existence of a "kinetic depinning field" which is lower than the static depinning field; that is, the field under which a moving DW can pass a trap may be lower than the field required to depin a DW which is initially in an equilibrium pinned configuration at the trap. The existence of distinct static and kinetic depinning fields for viscous DW motion (below the Walker field) has been demonstrated in simulations ${ }^{6}$ and investigated experimentally. ${ }^{7}$ Kinetic depinning has also been observed indirectly as a "dynamical pinning" effect, ${ }^{8,9}$ which occurs when the Walker field lies in between the static and kinetic pinning fields of a random potential due to edge roughness. In this paper we study the situation where the DW motion takes place above the Walker field and the DW interacts with a single, welldefined pinning site. We show experimentally that distinct static and kinetic depinning fields also exist in this case, with the oscillatory nature of the DW motion leading to a distribution of kinetic depinning fields. Micromagnetic simulations are in good qualitative agreement with our results.

In this paper, we study the interaction between a transverse DW (Ref. 10) and a T-shaped trap. ${ }^{11}$ The two possible equilibrium pinned configurations for a head-to-head DW are shown in Fig. 1(a): the trap acts either as a potential well or a potential barrier, depending on the orientation of the magnetization in the core of the DW, and the two equilibrium configurations can be clearly distinguished by measuring the depinning field. In order to measure the static and kinetic pinning fields, we fabricated $100 \mathrm{~nm}$ wide and $10 \mathrm{~nm}$ thick L-shaped nanostrips with T-shaped traps at a distance of $20 \mu \mathrm{m}$ from the corner of the L-shape [Fig. 1(b)], using electron beam lithography, thermal evaporation of Permalloy

${ }^{\text {a)} E l e c t r o n i c ~ m a i l: ~ e . l e w i s 06 @ i m p e r i a l . a c . u k . ~}$
$(\mathrm{NiFe})$, and a lift-off process. Global magnetic fields are applied to the sample in two ways: slowly varying fields are applied via an external quadrupole electromagnet, and fast field pulses are applied (to the whole nanostructure) using current pulses in a $300 \mu \mathrm{m}$ wide stripline on the back of the $50 \mu \mathrm{m}$ thick Si substrate. Magnetization switching of the structures was measured using a magneto-optical Kerr effect magnetometer (MOKE) with a laser spot diameter of $\sim 5 \mu \mathrm{m}$; the spot is positioned after the trap [dashed ellipse in Fig. 1(b)]. The setup is described in more detail in Ref. 12. The experiment is described schematically in Figs. 1(c) and 1(d). The structure is initially magnetized along $+x$ on the horizontal arm and along $-y$ in the vertical arms [c(i), d(i)]. In order to measure the kinetic pinning field [Fig. 1(c)], a saturating field is then applied along $(-1,-1)$ to create a DW at the corner of the nanostructure and switch the magnetization in the horizontal arm from $+x$ to $-x$ [c(ii)]. A constant field along $+x$ of $\sim 50$ Oe is then applied, which is below the depinning field from the corner [c(iii)]. (The corner depinning field is $\sim 70-75$ Oe. The outer radius of curvature of the corner is $\sim 120 \mathrm{~nm}$, which gives rise to this
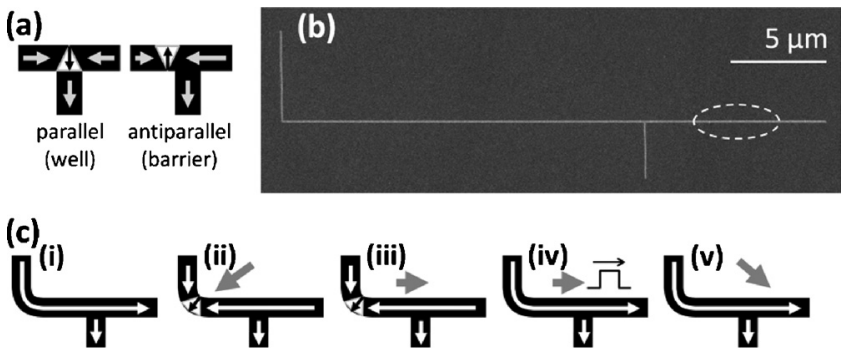

(d) (i)

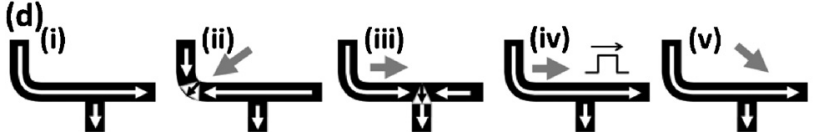

FIG. 1. Measuring the static and kinetic pinning fields at a T-shaped trap. (a) Equilibrium pinned configurations for a head-to-head DW at a T-shaped trap. The T-shape acts as a potential well when the magnetization in the core of the DW is parallel to the magnetization in the transverse arm and as a potential barrier when the magnetization is antiparallel. (b) The SEM image of an L-shaped nanostructure with a trap. The position of the MOKE laser spot is indicated by the dashed ellipse. (c) Schematic of kinetic depinning measurement, showing magnetization configurations and applied fields. The thicker, gray arrows indicate the fields applied using the electromagnet and the thinner, black arrows indicate the fast field pulse applied via the stripline. (d) Schematic of static depinning measurement. 


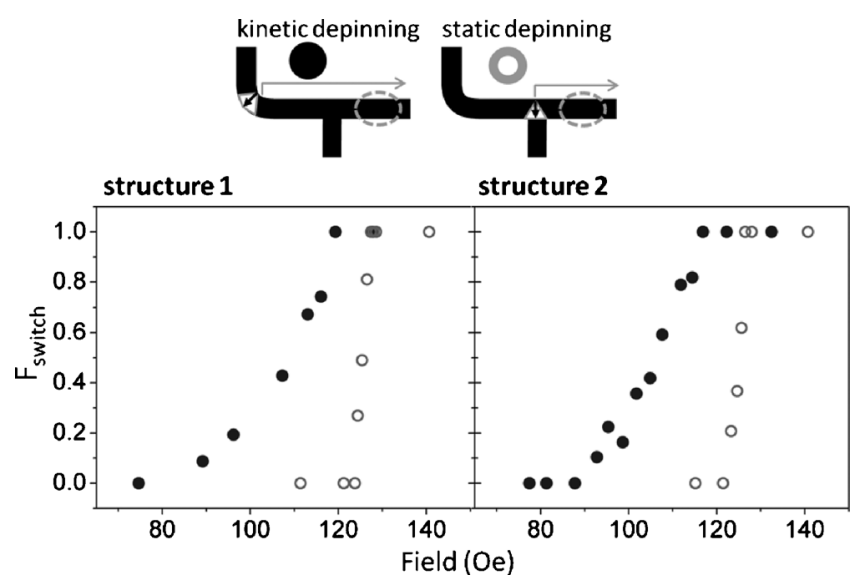

FIG. 2. Static and kinetic depinning fields at a T-shaped trap. The plots show the switching fraction $\mathrm{F}_{\text {switch }}$ as a function of field, measured on two nominally identical structures. The quoted field is the total field applied during the pulse, i.e., the sum of the fields applied via the stripline and via the electromagnet. Filled circles: $\mathrm{F}_{\text {switch }}$ measured when the DW is initially pinned at the corner, corresponding to kinetic depinning of the DW as it passes through the T-shaped trap. Open circles: $\mathrm{F}_{\text {switch }}$ measured when the DW is initially pinned at the trap, corresponding to static depinning of the DW from the trap. The dashed ellipses indicate the position of the MOKE laser spot.

relatively high depinning field. $)^{13}$ A $5.2 \mu$ s field pulse is subsequently applied via the stripline to depin the DW from the corner and drive it toward the trap. The risetime of the pulse is much shorter than the time taken for the DW to travel to the trap, ${ }^{14}$ so the field under which the moving DW interacts with the trap is well-defined. Figure 1(c)(iv) indicates the resulting configuration when the DW passes through the trap and annihilates at the end of the strip. Finally, a saturating field is applied along $(1,-1)[\mathrm{c}(\mathrm{v})]$ to sweep any remaining DW out of the structure and set the magnetization along $+x$.

The corresponding measurement for the static case is shown in Fig. 1(d). Here, the constant field along $x$ that is applied in stages (iii) and (iv) is 80 Oe (rather than 50 Oe as for the kinetic measurement). This 80 Oe field is above the depinning field from the corner, but below the depinning field from the trap, so that in step (iii) the DW moves to the trap and is pinned there. A $5 \mu$ s pulse is then applied to depin the DW from the trap. In both the static and the kinetic case, the measurement is repeated 100 times to obtain the probability of switching on application of the pulse. The switching probability is given by the height of the step in the averaged MOKE signal on application of the pulse [stages (iii) and (iv)], normalized to the height of the step corresponding to switching between fully saturated states [stages (i) and (ii)].

The probability of switching on application of the fast field pulse, $F_{\text {switch }}$, is plotted as a function of field in Fig. 2 for two separate structures. The filled circles correspond to the measurement sequence shown in Fig. 1(c), i.e., kinetic depinning of the DW from the trap. (We also checked that the DW does depin from the corner, by positioning the MOKE spot between the corner and the trap; the DW was found to always depin from the corner for fields $>75$ Oe.) The open circles correspond to the situation where the DW is initially pinned at the trap, i.e., the static depinning case [Fig. 1(d)]. This switching distribution is well-separated from the kinetic pinning fields; switching occurs at $\sim 125$ Oe in the static case, while in the kinetic case the switching field distribution extends over the range 90-115 Oe. Figure 2 thus demonstrates the existence of distinct static and kinetic pinning fields.

The step in $\mathrm{F}_{\text {switch }}$ corresponding to kinetic depinning is wider than that corresponding to static depinning. In the static case, the DW is initially in an equilibrium pinned configuration at the trap, and the finite width of the step is attributed to the thermally activated nature of the depinning. In the kinetic case, the DW is initially moving under an applied field well above the Walker field of $\sim 17$ Oe. ${ }^{12}$ In this higher field regime, the DW continually transforms between the two possible transverse configurations via the nucleation of an antivortex which crosses the strip. ${ }^{5,15}$ There are many possible states in which the DW can reach the trap. Although the same field sequence is applied on each of the 100 repetitions, the DW is not expected to reach the trap in the same state every time. The spatial period of the motion in the range $60-130$ Oe has been simulated as $\sim 200 \mathrm{~nm}$, so the DW is expected to undergo approximately 100 cycles as it moves $20 \mu \mathrm{m}$ to the trap. Over such a large number of cycles, small variations in the field at which the DW depins and thermal fluctuations during its motion act to randomize the state in which the DW reaches the trap. The value of $F_{\text {switch }}$ corresponds to the fraction of the possible DW configurations which are able to pass through the trap. As the field is increased, a larger fraction of the possible configurations are able to pass the trap, giving rise to the observed distribution of depinning fields. This is different to the situation where the DW is moving below the Walker threshold. Pinning sites with pinning fields below the Walker field have a single kinetic depinning field (not a distribution of fields), because there is only one possible state for the moving DW. This single kinetic pinning field has been calculated analytically for a constant-gradient barrier.

The data of Fig. 2 show not only that the dynamical state of the DW affects the DW pinning, but also that the pinning site affects the final state of the DW. This can be seen by considering the equilibrium pinned configurations shown in Fig. 1(a). In the parallel case [Fig. 1(a)], the DW will depin at the static depinning field of $\sim 125 \mathrm{Oe}$, while in the antiparallel case the DW is blocked in front of the trap and no switching occurs until the nucleation field of $\sim 250$ Oe is applied. In our static depinning experiment, the DW travels to the trap under a field of $\sim 70-75$ Oe (the depinning field from the corner) and relaxes to an equilibrium pinned configuration at the trap. It might be expected that the DW should sometimes become pinned in the antiparallel configuration, because the motion of the DW as it travels toward the trap involves repeated transformations between the two possible transverse DW types. This is not observed: the static depinning probability $\mathrm{F}_{\text {switch }}$ saturates at 1 , even though the maximum field applied to depin the DW is $140 \mathrm{Oe}$, well below the antiparallel switching field of 250 Oe. In other words, the DW appears to be always pinned in the parallel configuration. In the kinetic case, $\mathrm{F}_{\text {switch }}$ again saturates at 1; there is no evidence of the DW becoming blocked in the antiparallel configuration. These results are discussed in light of micromagnetic simulations below.

We used the OOMMF package ${ }^{16}$ to simulate the DW-trap interaction; the simulation parameters were $\mathrm{M}_{\mathrm{s}}$ $=800 \mathrm{kA} \mathrm{m}^{-1}$, exchange stiffness $\mathrm{A}=13 \times 10^{-12} \mathrm{~J} \mathrm{~m}^{-1}$, and damping constant $\alpha=0.01$. The cell size was $3.5 \times 3.5$ 
(a)
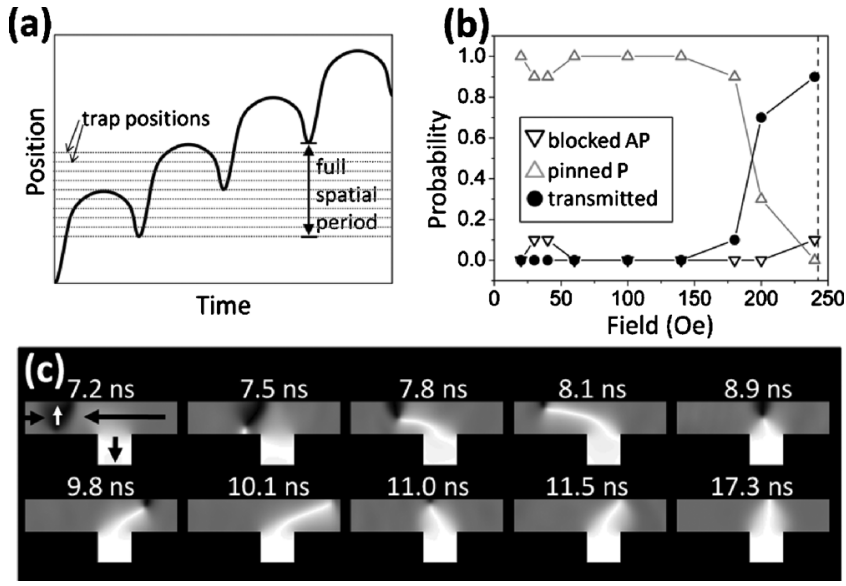

FIG. 3. Micromagnetic simulations. (a) Typical simulated position-time plot for a moving DW, with gray lines indicating the ten trap positions. (b) Probability of each final DW state-blocked AP, pinned $\mathrm{P}$, or transmitted-as a function of field. The solid lines are guides to the eyes. The dashed line indicates the simulated static depinning field for a DW pinned in the parallel configuration. (c) Snapshots of the simulated DW interaction for one of the trap positions. The interaction with the T-shape causes the DW to become pinned in the parallel configuration. The applied field is $60 \mathrm{Oe}$. The grayscale indicates the $y$-component of the magnetization, where the scale runs from white $(-y)$ to black $(+y)$.

$\times 10 \mathrm{~nm}^{3}$. We first performed simulations on plain nanostrips in order to determine the (field-dependent) spatial period of the motion. For each different field value, we then performed ten separate simulations with a T-shaped trap at one of ten different positions; the position increment was one-tenth of the full spatial period of the DW motion. This is described schematically in Fig. 3(a). The results of the simulations are summarized in Fig. 3(b), which shows the probability of each final DW state (blocked in front of the trap in the antiparallel configuration, pinned inside the trap in the parallel configuration, or transmitted) as a function of field. The probability of a particular state represents the fraction of the ten measured positions which resulted in that state. The dashed line indicates the simulated static depinning field. Figure 3(b) shows that the T-shaped trap has distinct simulated static and kinetic pinning fields, because nonzero values of the transmission probability (filled circles) are observed for fields below the simulated static depinning field (dashed line). Qualitatively, the behavior is very similar to that observed experimentally, if we associate the simulated transmission probability (filled circles) with the experimental kinetic depinning measurements (filled circles on Fig. 2). (This corresponds to assuming that the DW phase is fully randomized in the experiment.) The transmission probability increases gradually over a range of fields below the static depinning field, consistent with the experimental distribution of kinetic depinning fields. In addition, the DW very rarely becomes blocked in the antiparallel configuration; instead, the final state of the DW is either pinned in the parallel configuration or transmitted. This strong bias toward pinning in the parallel configuration rather than the antiparallel configuration is consistent with the experimental static depinning results: when the DW moves to the trap [stage (ii) and (iii) of Fig. 1(d)], it always ends up pinned in the parallel configuration, and the measured static depinning field always corresponds to depinning in this parallel configuration. (Note that simulations of the dynamical interaction with a crossshaped trap of the type used in previous work ${ }^{17}$ showed different behavior: for a cross shaped trap, the bias toward pinning in the parallel configuration is weaker and there is an increased probability of blocking in the antiparallel configuration.) Snapshots of the simulated interaction between the DW and the trap [Fig. 3(c)] show how the interaction with the trap can act to distort the structure of the moving DW, resulting in a final state where the DW is pinned in the parallel configuration. Altogether, the simulations are in good qualitative agreement with the experiments, although the degree of randomization of the DW phase in the experiment is not accurately known. Quantitatively, the experiments and simulations differ in that the depinning occurs at higher fields in the simulations than in the experiments. In the static case, this is attributed to the thermally activated nature of the depinning, ${ }^{18}$ but more work is needed to elucidate the role of thermal effects in the kinetic depinning process.

In conclusion, we have demonstrated the existence of distinct static and kinetic pinning fields for DW motion above the Walker threshold. The oscillations of the DW structure lead to a distribution of kinetic pinning fields. Our experimental results are in good qualitative agreement with the results of micromagnetic simulations.

The work and results reported in this letter were obtained with research funding from the European Community under the Seventh Framework Programme Contract No. 247368:3SPIN.

${ }^{1}$ S. S. P. Parkin, M. Hayashi, and L. Thomas, Science 320, 190 (2008).

${ }^{2}$ D. A. Allwood, G. Xiong, C. C. Faulkner, D. Atkinson, D. Petit, and R. P. Cowburn, Science 309, 1688 (2005).

${ }^{3}$ M. Diegel, S. Glathe, R. Mattheis, M. Scherzinger, and E. Halder, IEEE Trans. Magn. 45, 3792 (2009).

${ }^{4}$ M. Donolato, P. Vavassori, M. Gobbi, M. Deryabina, M. F. Hansen, V. Metlushko, B. Ilic, M. Cantoni, D. Petti, S. Brivio, and R. Bertacco, Adv. Mater. (Weinheim, Ger.) 22, 2706 (2010).

${ }^{5}$ A. Thiaville and Y. Nakatani, Spin Dynamics in Confined Magnetic Structures III (Springer, Berlin, 2006).

${ }^{6}$ S. M. Ahn, D. H. Kim, and S. B. Choel, IEEE Trans. Magn. 45, 2478 (2009).

${ }^{7}$ S. M. Ahn, K. W. Moon, D. H. Kim, and S. B. Choe, Appl. Phys. Lett. 95, 152506 (2009).

${ }^{8}$ H. Tanigawa, T. Koyama, M. Bartkowiak, S. Kasai, K. Kobayashi, T. Ono, and Y. Nakatani, Phys. Rev. Lett. 101, 207203 (2008).

${ }^{9}$ X. Jiang, L. Thomas, R. Moriya, M. Hayashi, B. Bergman, C. Rettner, and S. Parkin, Nature Communications 1, 25 (2010).

${ }^{10}$ R. D. McMichael and M. J. Donahue, IEEE Trans. Magn. 33, 4167 (1997).

${ }^{11}$ D. Petit, A. V. Jausovec, H. T. Zeng, E. Lewis, L. O’Brien, D. Read, and R. P. Cowburn, Phys. Rev. B 79, 214405 (2009).

${ }^{12}$ E. R. Lewis, D. Petit, L. O’Brien, A. Fernandez-Pacheco, J. Sampaio, A. V. Jausovec, H. T. Zeng, D. E. Read, and R. P. Cowburn, Nature Mater. 9, 980 (2010).

${ }^{13}$ E. R. Lewis, D. Petit, L. Thevenard, A. V. Jausovec, L. O'Brien, D. E. Read, and R. P. Cowburn, Appl. Phys. Lett. 95, 152505 (2009).

${ }^{14} \mathrm{The}$ pulse risetime is $15 \mathrm{~ns}$. The DW velocity in the range $60-130$ Oe is less than $100 \mathrm{~m} \mathrm{~s}^{-1}$ (see Ref. 12), corresponding to a minimum travel time over $20 \mu \mathrm{m}$ (from the corner to the trap) of $200 \mathrm{~ns}$.

${ }^{15}$ Y. Nakatani, A. Thiaville, and J. Miltat, Nature Mater. 2, 521 (2003).

${ }^{16}$ The oOMMF code and user guide is available at http://math.nist.gov/ oommf.

${ }^{17}$ E. R. Lewis, D. Petit, A. V. Jausovec, L. O’Brien, D. E. Read, H. T. Zeng, and R. P. Cowburn, Phys. Rev. Lett. 102, 057209 (2009).

${ }^{18}$ A. Himeno, T. Okuno, T. Ono, K. Mibu, S. Nasu, and T. Shinjo, J. Magn. Magn. Mater. 286, 167 (2005). 